\title{
Parálisis periódica tirotóxica
}

\section{Thyrotoxic periodic paralysis}

\author{
Jorge Ludwing Duarte, Álvaro Sanabria \\ - Bucaramanga (Colombia)
}

\section{Resumen}

Presentamos el caso de un hombre de 39 años quien sufrió dos episodios de parálisis por hipocaliemia asociado a hipertiroidismo. Esta entidad inusual y considerada casi exclusiva de los países asiáticos también ha sido documentada en países occidentales; por tanto, damos a conocer este caso para ser tenido en cuenta entre los diagnósticos diferenciales en los servicios de urgencias (Acta Med Colomb 2011; 36: 145-148).

Palabras clave: parálisis periódica hipocaliémica tirotóxica, hipertiroidismo.

\section{Abstract}

We recorded the case of a 39 year-old man who suffered two episodes of paralysis and hipokalemia associated with hyperthyroidism. This unusual entity, almost exclusively of Asian countries, has been documented in western countries; therefore, we present this case to be considered in the differential diagnosis in emergency departments (Acta Med Colomb 2011; 36: 145-148).

Key words: thyrotoxic hypokalemic periodic paralysis, hyperthyroidism.

Dr. Jorge Ludwing Duarte Cala: Endocrinólogo, Universidad Nacional de Colombia, Profesor de Cátedra del Departamento de Medicina Interna, Universidad Industrial de Santander, Endocrinólogo Hospital Universitario de Santander; Dr. Álvaro Sanabria Naranjo: Residente de Medicina Interna, Universidad Industrial de Santander, Hospital Universitario de Santander. Bucaramanga (Colombia).

Correspondencia: Dr. Alvaro Sanabria Naranjo. Bucaramanga (Colombia).

E-mail: alvarosanabria80@hotmail.com Recibido: 22/III/2011 Aceptado: 14/VII/2011

\section{Descripción del caso}

Reportamos el caso de un hombre de 39 años de raza mestiza con antecedente de asma, consumidor de bebidas alcohólicas mensual desde hace 15 años, sin antecedentes familiares de importancia; consulta de urgencia por la incapacidad de mover las piernas desde las 21:45 horas al intentar bajarse de su automóvil después de manejar por 10 horas, sufre parestesias, posterior paraparesia y evoluciona a cuadriparesia rápidamente. Negó episodios similares previos, síntomas gripales, diarrea, vómito, fiebre o trauma. Desde hace un mes ha notado pérdida de peso ( $4 \mathrm{~kg}$ ) no justificada por dieta ni ejercicio, además ocho días de temblor distal.

Ingresa al servicio de urgencias en silla de ruedas, alerta, con tensión arterial de 140/60 mmHg, frecuencia cardiaca de
100 , frecuencia respiratoria de 18 y temperatura de $36.5^{\circ} \mathrm{C}$, pesó $70 \mathrm{~kg}$, fuerza muscular de $1 / 5$ en miembros inferiores, 2/5 en miembros superiores, respetando los músculos de la respiración, sin compromiso sensitivo, ni esfinteriano, reflejos osteotendinosos rotulianos ausentes, pares craneales normales, tenía un bocio grado 2 , sin exoftalmos; el examen de tórax y abdomen fue normal. Se consideró inicialmente una polineuropatía tipo Guillain Barré, los primeros laboratorios fueron tomados nueve horas de iniciado los síntomas (Tabla 1). El EKG mostró un ritmo sinusal con un moderado aplanamiento de la onda $\mathrm{T}$. El paciente es hospitalizado en observación con reposo y líquidos endovenosos tipo lactato ringer sin reposición de potasio desde su ingreso, con recuperación completa de la debilidad muscular en

Tabla 1. Laboratorios tomados nueve horas después del primer episodio.

\begin{tabular}{|c|c|c|c|c|c|}
\hline Calcio iónico & $4,21 \mathrm{mg} / \mathrm{dL}$ & $\mathrm{Hb}$ & $12.3 \mathrm{~g} / \mathrm{dL}$ & PT & 15 seg INR 1.12 \\
\hline Cloro & $103.4 \mathrm{mEq} / \mathrm{L}$ & Htco & $37.1 \%$ & PTT & $24.8 \mathrm{seg}$ \\
\hline Sodio & $141.7 \mathrm{mEq} / \mathrm{L}$ & Leucocitos & $6300 / \mathrm{mm}^{3}$ & Creatinina & $0.84 \mathrm{mg} / \mathrm{dL}$ \\
\hline Potasio & $4.08 \mathrm{mEq} / \mathrm{L}$ & Neutrófilos & $79 \%$ & BUN & $12.91 \mathrm{mg} / \mathrm{dL}$ \\
\hline $\mathrm{CK}$ & $2167 \mathrm{U} / \mathrm{L}$ & Linfocitos & $19 \%$ & ASAT & $58.4 \mathrm{U} / \mathrm{L}$ \\
\hline Elisa VIH & Negativo & Monocitos & $2 \%$ & ALAT & $82.6 \mathrm{U} / \mathrm{L}$ \\
\hline TSH & $<0.05 \mathrm{mU} / \mathrm{L}$ & Plaquetas & $179.000 / \mathrm{mm}^{3}$ & Serología & 0 diluciones \\
\hline
\end{tabular}


las próximas 14 horas, se hizo diagnóstico presuntivo de episodio de parálisis hipocalémica tirotóxica, y es remitido para valoración por endocrinología.

El potasio en orina de 24 horas fue de $15.18 \mathrm{mEq} / \mathrm{L}$ (20-80mEq/L). Endocrinología hace diagnóstico de enfermedad de Graves, inicia corticosteroides, betabloqueador y posterior tratamiento con yodo radioactivo.

Veintiun días después (aún sin recibir yodoterapia) presenta nuevo episodio de debilidad muscular a la 1 a.m. mientras dormía y después de varias horas de conducir en el día, con evolución a cuadriparesia, y un episodio de emesis escasa posterior a la parálisis, sin afección de los músculos respiratorios, ingresa de nuevo al hospital con TA de 120/50 mmHg, Fc: 100/min, Fr: 19/min y afebril. Se toman laboratorios seis horas después de iniciados los síntomas, con potasio $1.46 \mathrm{mEq} / \mathrm{L}$, sodio $143 \mathrm{mEq} / \mathrm{L}$, cloro $105 \mathrm{mEq} / \mathrm{L}$, creatinina $0.97 \mathrm{mg} / \mathrm{dL}, \mathrm{Hb} 13.8 \mathrm{~g} / \mathrm{dL}$, leucocitos 10000 , neutrófilos $86 \%$, linfocitos $10 \%$ y plaquetas 205000 , $\mathrm{TSH}<0.05 \mathrm{mU} / \mathrm{L}$, T4 libre $41.8 \mathrm{pmol} / \mathrm{L}$ y T3 $3.95 \mathrm{nmol} / \mathrm{L}$, $\mathrm{pH} 7.41, \mathrm{pO}_{2} 86.3 \mathrm{mmHg}, \mathrm{pCO}_{2} 31.9 \mathrm{mmHg}, \mathrm{HCO}_{3} 20.2$ $\mathrm{mEq} / \mathrm{L}, \mathrm{SO}_{2} 96.6 \%$. El paciente recupera la fuerza muscular 18 horas de iniciado los síntomas, con potasio de 4.02 $\mathrm{mEq} / \mathrm{L}, \mathrm{y}$ es dado de alta con betabloqueador, durante este segundo episodio fue tratado con solución salina normal y no recibió reposición de potasio. Posteriormente recibió dosis de yodo radioactivo $\mathrm{I}-131(30 \mathrm{mCi})$ y actualmente tiene 10 meses sin episodios de parálisis, cesó el temblor y aumentó de peso; con un T4 libre de control de 2.39 pmol/L se inició tratamiento con levotiroxina.

\section{Discusión}

La parálisis por hipocaliemia periódica tirotóxica o parálisis periódica tirotóxica (PPT), es una alteración neuromuscular poco frecuente caracterizada por episodios de debilidad recurrente debido a niveles de potasio bajo, secundario a exceso de la hormona tiroidea (1), y que en ocasiones puede ser mortal (2).

La PPT fue descubierta por Rosenfeld en 1902 (3), es una de las causas de parálisis por hipocaliemia prevalente en personas con hipertiroidismo en oriente, ocasionada por la redistribución del potasio del espacio extracelular al intracelular, y es un diagnóstico diferencial de la parálisis por hipocaliemia periódica familiar en países occidentales. Es importante que recordemos causas de parálisis por hipocaliemia no periódica debido a pérdida de potasio como resultado de pérdidas gastrointestinales, uso de diuréticos, hiperaldosteronismo primario, abuso en el consumo de bebidas alcohólicas, abuso de tolueno, acidosis tubular renal, síndrome de Bartter y Gitelman (4).

La incidencia de PPT en las personas con hipertiroidismo es de 1.8-8.8\% y algunos reportan hasta $20 \%$ dependiendo del país asiático, y del $0.1-0.2 \%$ en Estados Unidos $(2,5,6,8,9)$. Es más frecuente en hombres asiáticos y blancos con una relación de 11-70:1 con respecto a mujeres, y entre la segunda a quinta década de vida $(5,6,10)$; raramente se presenta en la adolescencia (la parálisis familiar por hipocaliemia es más frecuente en menores de 20 años) (2). Aun así la incidencia de la enfermedad ha disminuido en el oriente en los últimos 50 años a causa de cambios en la dieta, menor consumo de carbohidratos y mayor consumo de potasio $(2,6)$. Por otro lado se considera que ha aumentado la incidencia de la PPT en países del occidente, probablemente por cruces raciales y a la facilidad actual para el traslado de una región a otra (11), teniendo registros en 74 reportes de casos de personas no asiáticas entre 1986 y 2005 (2), como lo ha registrado Perú (12), aunque este paciente tenía descendencia asiática. El caso que presentamos es de nacionalidad colombiana sin ascendencia asiática; no encontramos casos registrados en nuestro país.

La presentación clínica de la PPT es la de episodios de debilidad muscular temprano en la mañana, incluso con imposibilidad para levantarse de la cama (1), después de un descanso prolongado y posterior a la realización de una actividad física extenuante, $\mathrm{o}$ al haber ingerido una cantidad de alimentos rica en carbohidratos (2), se presenta como debilidad leve, generalizada y asimétrica de predominio en miembros inferiores y músculos proximales, aunque puede llegar a debilidad severa con compromiso de músculos respiratorios, oculares y de la deglución $(2,8,13,14)$. Otros factores desencadenantes y menos frecuentes son las infecciones, trauma, la menstruación, y el frío (10). Aunque la tendencia de la parálisis es hacia la remisión en las horas siguientes al inicio del cuadro clínico hasta dos a cuatro días $(5,6,9,15)$ puede llevar a un desenlace mortal debido al compromiso cardiovascular, o a la acidosis respiratoria o por rabdomiólisis $(15 \%)(10,13)$; la creatinfosfoquinasa se eleva en cerca de $66 \%$ de los casos, más aun si realizó un ejercicio previo (2). La parálisis suele presentarse con el inicio del hipertiroidismo, con escasos o ningún síntoma previo (7-9) y algunos encuentran relación con meses calurosos (6).

La hipocaliemia de los pacientes con PPT por lo general es menor de $3.0 \mathrm{mEq} / \mathrm{L}$ y se presenta por la entrada del potasio a las células, favorecido por un estímulo adrenérgico sobre la bomba sodio-potasio ATPasa en músculos, hígado y riñones $(2,7,16)$ incluso mayor que el de otras personas hipertiroideas, de ahí el poder benéfico de los betabloqueadores para el control de la PPT $(7,15)$. Además hay un aumento de la insulina circulante (2) e hipofosfatemia. Los estrógenos se relacionan con una baja actividad de la bomba sodio-potasio ATPasa, de ahí es posible la presentación menos frecuenta de la PPT en mujeres $(10,17)$. Algunos polimorfismos de nucleótidos simples del gen CACNA1S (codifica la subunidad del canal de calcio tipo L) evaluados en parálisis por hipocaliemia periódica familiar y algunos HLA DRw8, A2, Bw22; Aw19, B17, B5, y Bw46 han sido encontrados en algunos asiáticos con PPT (16); sin embargo, ésta predisposición no es necesaria para que se presente el evento, y una causa genética y autoinmune única no ha sido establecida $(2,14)$. 
Para realizar el diagnóstico es necesario tener en cuenta la historia clínica personal y familiar (para detectar casos de parálisis periódica familiar), indagar sobre síntomas sutiles de hipertiroidismo, realizar pruebas de función tiroidea, en la que la TSH se encuentra suprimida con T4 libre elevada, siendo así la enfermedad de Graves la causa más frecuente $(3,6)$. La PPT podría ser la manifestación de un hipertiroidismo secundario, o estar asociada a medicamentos como la amiodarona (14), adrenalina, glucocorticoides a altas dosis, fisostigmina, interferón alfa en el tratamiento de hepatitis C (17), o por un hipertiroidismo facticio (18) o uso de antiretrovirales (14). Se ha encontrado también en tumores de la pituitaria productor de tirotropina (19), por adenoma tiroideo tóxico y tiroiditis subaguda $(6,3)$.

Los hallazgos en los niveles de fósforo, en el estado ácido base, la excreción de potasio en orina de 24 horas, y en el electrocardiograma ayudan a acercarnos al diagnóstico de esta entidad y de sus diagnósticos diferenciales $(4,6,8,9)$. En nuestro caso la excreción de potasio en orina de 24 horas nos muestra que no existe pérdidas renales de este electrolito. El nivel de potasio en los pacientes con PPT durante el episodio agudo con compromiso respiratorio suele ser menor de $2.0 \mathrm{mmol} / \mathrm{L} \mathrm{(16).} \mathrm{La} \mathrm{taquicardia,} \mathrm{la} \mathrm{hipertensión} \mathrm{arterial}$ sistólica, el estado ácido-base normal y la hipofosfatemia $(66 \%)$ es característico de la PPT $(2,7,20)$. El diagnóstico de esta entidad puede ser pasado por alto o erróneamente diagnosticado, provocando así episodios recurrentes de la parálisis, con una severidad que hasta el momento no puede ser predecible (2), sin contar el uso de tratamientos no apropiados como la acetazolamida usado para la parálisis por hipocaliemia familiar y que puede predisponer la parálisis en los pacientes con PPT $(2,3)$.

El electrocardiograma de los pacientes con PPT se caracteriza por un voltaje alto del QRS y bloqueo AV de primer grado con una especificidad de más de $85 \%$ pero menor en su sensibilidad, signos asociados también a la hipocaliemia (4).

La electromiografía muestra un compromiso miopático que desaparece durante la remisión de la debilidad (6), en la biopsia muscular hay anormalidad en $75 \%$ de los casos con atrofia, proliferación nuclear, vacuolización, entre otros, pero en la microscopía se observa además compromiso mitocondrial y granulaciones de glucógeno en todas las fibras musculares, hallazgos también vistos en captación con $\mathrm{Tc} 99 \mathrm{~m}(6,21)$.

La debilidad muscular suele mejorar sin tratamiento y al tiempo que los niveles de potasio y fósforo se normalizan, no hay consenso con cuál valor de potasio debe iniciarse la reposición, teniendo en cuenta que es un problema de redistribución y por tanto puede corregirse sin necesidad de aportar potasio; además cerca de 40- 63\% sufren hipercaliemia por rebote, más aún si se administran $>90$ meq de $\mathrm{KCl}$ al día $(7,8,20)$. Sin embargo, los trastornos de conducción cardiaca son un problema que amerita reponer de manera oportuna este electrolito. La reposición recomendada es de menos de $10 \mathrm{mEq} / \mathrm{h}$ de acuerdo con si tiene o no anormalidad electrocardiográfica, se mantendrá la reposición hasta mejorar la debilidad muscular (15). Se ha encontrado que los niveles de fósforo llegan a niveles normales después del tratamiento con potasio (6).

Los episodios de parálisis cesan al mantener el paciente en estado eutiroideo por medio de medicamentos antitiroideos, ablación con yodo-radioactivo, o con cirugía o al eliminar la causa secundaria $(3,10,14)$, sin embargo los síntomas pueden reaparecer días a dos semanas después de realizar el tratamiento quirúrgico o con yodo (13). El uso de betabloqueadores no selectivos (propanolol) reduce los episodios y la severidad de la parálisis durante el episodio agudo a $20-40 / \mathrm{mg}$ vía oral cada ocho horas con el sustento de minimizar el efecto adrenérgico $(2,3,6,18)$. Mientras se realiza el tratamiento definitivo se recomienda además del betabloqueador, el evitar los factores desencadenantes como el ejercicio extenuante, alimentos ricos en carbohidratos, consumo de bebidas alcohólicas y dieta alta en sal (9).

El uso de potasio oral de manera ambulatoria no ha demostrado reducir los ataques de parálisis (14). La espironolactona ha dado resultado en algunos casos aislados, en los cuales han continuado en un plan de tratamiento antitiroideo (17).

La incidencia de la PPT aunque es una entidad rara en personas no asiáticas, ha aumentado en países del occidente (2) y debemos tenerla en cuenta entre los diagnósticos diferenciales de la debilidad muscular en urgencias.

\section{Referencias}

1. Radulescu D, Parv A, Pripon S, Radulescu ML, Gulei I, Buzoianu A. Hypokalemic periodic paralysis in hyperthyroidism - rare event. Endocrinologist 2010; 20: 72-4.

2. Kung AWC. Clinical review: thyrotoxic periodic paralysis: a diagnostic challenge J Clin Endocrinol Metab 2006; 91: 2490-5.

3. Kokenge F, Moening $\mathbf{H}$. Thyrotoxic periodic paralysis: a peculiar case with weekend-related quadriplegia. Endocrinologist 2005; 15: 297-9.

4. Hsu YJ, Lin YF, Chau T, Liou JT, Kuo SW, Lin SH. Electrocardiographic manifestations in patients with thyrotoxic periodic paralysis. Am J Med Sci 2003; 326: $128-32$

5. Cesur M, Ilgin SD, Baskal N, Gullu S. Hypokalemic paralysis is no just a hypolemic paralysis. Eur J Emerg Med 2008; 15: 150-153.

6. Magsino CH, Ryan J. Thyrotoxic periodic paralysis. South Med J 2000; 93: 996-1003.

7. Lin YF, Wu CC, Pei D, Chu SJ, Lin SH. Diagnosing thyrotoxic periodic paralysis in the ED. Am J Emerg Med 2003; 21: 339-42.

8. Liu YC, Tsai WS, Chau T, Lin SH. Acute hypercapnic respiratory failure due to thyrotoxic periodic paralysis. Am J Med Sci 2004; 327(5): 264-7.

9. Lam Lien, Nair RJ, Tingle L. Thyrotoxic periodic paralysis. Proc (Bayl Univ Med Cent). 2006; 19:126-9.

10. Abbasi B, Sharif Z, Sprabery LR. Hypokalemic thyrotoxic periodic paralysis eith thyrotoxic psychosis and hypercapnic respiratory failure. Am J Med Sci 2010; 340(2): $147-53$.

11. Perez E, Courville K. Parálisis periódica tirotóxica. Presentación de un caso. Revista Médico Científica Panamá; 15(1): 43-6.

12. Añaños Perez A, Díaz F. Paralisis periódica tirotóxica hipocalemica. Bol Soc Peru Med Interna 1996; 9(3): 106-8.

13. Celebi-Onder S, Dower JM, Jackson TK. Thyrotoxic periodic paralysis in 2 white men after treatment for hyperthyroidism with thyroidectomy and radioactive iodine. Endocrinologist 2007; 17: 255-7.

14. Barahona MJ, Vinagre I, Sojo L, Cubero JM, Perez A. Thyrotoxic periodic 
paralysis: a case report ant literatura review. $C M \& R$ 2009; 7(3): 96-8.

15. Lu KC, Hsu YJ, Chiu JS, Hsu YD, Lin SH. Effects of potassium supplementation on the recovery of thyrotoxic periodic paralysis. Am J Emerg Med 2004: 22: 544-7.

16. Kung AWC, Lau KS, Fong GCY, Chan V.Association of novel single nucleotide polymorphism in the calcium channel $\alpha 1$ subunit gene $\left(\mathrm{Ca}_{v} 1.1\right)$ and thyrotoxic periodic paralysis. J Clin Endocrinol Metab 2004; 89: 1340-5.

17. Tran HA, Reeves GE. Hepatitis $C$ infection and thyrotoxic periodic paralysis - a novel use of an old drug. Am J Med Sci 2008; 336(6): 515-8.

18. Chou HK, Tsao YT, Lin SH. An unusual cause of thyrotoxic periodic paralysis:
Thiiodothyronine-containing weight reducing agents. Am J Med Sci 2009; 337(1): 71-3

19. Laroia ST, Zaw KM, Ganti AK, Newman W, Akinwande AO. AmiodaroneInduced thyrotoxicosis presenting as hypokalemic periodic paralysis. South Med $J$ 2002; 95:1326-8

20. Lien YHH. A new diagnostic test for an old diagnostic challenge: thyrotoxic periodic paralysis. Crit Care Med 2006; 34(12): 3053-4.

21. Chang YY, Lan MY, Wu HS, Huang SH, Chen SS, Liu JS. Decreased muscular radionuclide uptake in Tc-99m MIBI Scintigraphy During paralytic phase of thyrotoxic periodic paralysis. Clin Nucl Med 2008 2008; 33: 297-8. 